\title{
Laboreal
}

Volume 15 N$^{\circ} 2$ | 2019

Varia

\section{JACQUES LEPLAT : bibliografia seletiva}

Corinne Lespessailles, Céline Levecque, Aurélie Puybonnieux e Agathe Vuillermet

\section{OpenEdition}

\section{Journals}

\section{Edição electrónica}

URL: http://journals.openedition.org/laboreal/15431

DOI: 10.4000/laboreal.15431

ISSN: 1646-5237

\section{Editora}

Universidade do Porto

\section{Refêrencia eletrónica}

Corinne Lespessailles, Céline Levecque, Aurélie Puybonnieux e Agathe Vuillermet, « JACQuES LEPLAT bibliografia seletiva », Laboreal [Online], Volume 15 N² | 2019, posto online no dia 01 dezembro 2019 consultado o 24 setembro 2020. URL : http://journals.openedition.org/laboreal/15431 ; DOI : https:// doi.org/10.4000/laboreal.15431

Este documento foi criado de forma automática no dia 24 setembro 2020.

\section{(c) (†) (8)}

Laboreal está licenciado com uma Licença Creative Commons - Atribuição-NãoComercial 4.0 Internacional. 


\title{
JACQUES LEPLAT : bibliografia seletiva
}

\author{
Corinne Lespessailles, Céline Levecque, Aurélie Puybonnieux e Agathe \\ Vuillermet
}

1 Esta bibliografia foi confirmada pela Biblioteca Gay-Lussac e pelo Centre de documentation sur la formation et le travail du Cnam (Conservatoire national des arts et métiers) [Centro de documentação sobre a formação e o trabalho do Cnam] no quadro de um seminário organizado à volta da Obra de Jacques Leplat pelo Groupe de recherche et d'étude sur l'histoire du travail et de l'orientation (Greshto) [Grupo de investigação e estudo sobre a história do trabalho e da orientação] no dia 19 de abril de 2019 em Paris.

2 Para uma bibliografia mais exaustiva sobre os trabalhos de Jacques Leplat anteriores a 1993, reenviamos o leitor para a recensão realizada pela revista Le Travail humain (vol. 56, n 2-3, 1993, pp. 267-275). Citamos igualmente o artigo publicado em 2006 na revista Laboreal de Catarina Silva (Sobre a Psicologia Ergonómica de Jacques Leplat, vol. 2, $\mathrm{n}^{\circ} 2$, pp. 47-61).

\section{Entrevista com Jacques Leplat}

32005 - Cloutier, E. \& Gaudart, C. Entrevue guidée avec Jacques Leplat. Perspectives interdisciplinaires sur le travail et la santé, 7(1). http://dx.doi.org/10.4000/pistes.3250

\section{Obras e Direção de Obras}

41958 - Faverge, J.-M., Leplat, J. \& Guiguet, B. L'Adaptation de la machine à l'homme. Paris : Presses universitaires de France.

51960 - Dellenbach, J., Faverge, J.-M. \& Leplat, J. Quelques aspects du vieillissement en rapport avec l'ergonomie. Paris : Centre national de la recherche scientifique.

61963 - Piéron, H., Chocholle, R. \& Leplat, J. Traité de psychologie expérimentale. II, Sensation et motricité. Paris : Presses universitaires de France. 
71968 - Leplat, J. Attention et incertitude dans les travaux de surveillance et d'inspection. Paris : Dunod.

1969 - Leplat, J. L’Enseignement programmé. Paris : Sociétés d'éditions scientifiques.

1969 - Leplat, J., Kalsbeek, J. \& Schmidtke, H. Travail mental et automatisation : activités mentales, fatigue mentale et activités de contrôle et de surveillance dans l'industrie. Luxembourg : Service des publications des Communautés européennes.

1970 - Leplat, J, Énard, C. \& Weill-Fassina, A. La Formation par l'apprentissage : éléments de psychopédagogie. Paris : Presses universitaires de France.

1974 - Leplat, J. Les Accidents du travail. Paris : Presses universitaires de France.

1977 - Cuny, X., Leplat, J. Introduction à la psychologie du travail. Paris : Presses universitaires de France.

1980 - Leplat, J. La Psychologie ergonomique. Paris : Presses universitaires de France.

1985 - Leplat, J. Erreur humaine, fiabilité humaine dans le travail. Paris : Armand Colin.

1987 - Rasmussen, J., Duncan, K. D. \& Leplat, J. (éd.). New technology and human error. Chichester ; New York : J. Wiley.

1990 - Leplat, J. (dir.) \& Terssac, G. de (dir.), Cellier, J.-M. (collab.), Neboit, M. (collab.), Oudiz A. (collab.). Les Facteurs humains de la fiabilité dans les systèmes complexes. Marseille : Octares. (Coll. Ergonomie et gestion).

1992 - Leplat, J. (coord.). L'Analyse du travail en psychologie ergonomique : recueil de textes, tome 1 . Toulouse : Octares.

1993 - Leplat, J. (coord.). L'Analyse du travail en psychologie ergonomique : recueil de textes, tome 2 . Toulouse : Octarès.

1997 - Leplat, J. Regards sur l'activité en situation de travail : contribution à la psychologie ergonomique. Paris : Presses universitaires de France.

2000 - Leplat, J. L’Analyse psychologique de l'activité en ergonomie : aperçu sur son évolution, ses modèles et ses méthodes. Toulouse : Octarès.

2001 - Leplat, J. \& Montmollin, M. de. Les Compétences en ergonomie. Toulouse : Octarès.

2002 - Leplat, J. Psychologie de la formation : jalons et perspectives : choix de textes (1955-2002). Toulouse : Octarès.

2008 - Leplat, J. Repères pour l'analyse de l'activité en ergonomie. Paris : Presses universitaires de France.

2011 - Leplat, J. Mélanges ergonomiques : activité, compétence, erreur. Toulouse : Octarès.

\section{Estudos e relatórios}

[s.d.] - Leplat, J. Communications et codage dans le travail. Paris: Laboratoire de psychologie du travail de l'École pratique des hautes études.

1965 - Leplat, J. Introduction sur les travaux effectués en exécution du contrat sur les aspects psychologiques et psychopédagogiques de la formation des techniciens. Paris : Centre d'études et recherches psychotechniques. 
1965 - Leplat, J. L'Organisation temporelle de la transmission de l'information. Paris : Centre d'études et recherches psychotechniques.

1973 - Leplat, J.. Les Critères dans les études économiques de sécurité routière. Paris : Laboratoire de psychologie du travail de l'École pratique des hautes études.

1975 - Leplat, J.. Genèse des accidents et facteurs de risque. Paris : Laboratoire de psychologie du travail.

1975 - Leplat, J. La Notion d'application en psychologie du travail. Paris : Université Paris 5 [Thèse de doctorat]

1976 - Leplat, J. Les Facteurs déterminant la charge de travail : rapport introductif. Paris : Laboratoire de psychologie du travail.

1976 - Leplat, J. Recherches en cours et travaux publiés 1974-1976. Paris : Laboratoire de psychologie du travail.

1978 - Leplat, J. Le Contrôle du système d'arrêt d'urgence dans une centrale nucléaire : observations préalables d'une étude ergonomique. Paris: Conservatoire national des arts et métiers, Laboratoire de physiologie du travail et d'ergonomie.

1990 - Rasmussen, J., Brehmer, B., Montmollin, M. de \& Leplat, J. (éd.). Taxonomy for analysis of work domains. Proceedings of the first MOHAWC workshop, Liège, May 15-16, 1990. Roskilde, Denmark : Risø National Laboratory.

1992 - Brehmer, B. \& Leplat, J. (éd.). Simulations, evaluations and models. Proceedings of the fourth Mohawc Workshop, Bamberg, October 22-23, 1991. Roskilde, Denmark: Risø National Laboratory.

1996 - Leplat, J. À propos de l'environnement: questions terminologiques et théoriques. Paris : École pratique des hautes études.

\section{Contribuições em obras coletivas}

1969 - Leplat, J. Les liaisons sensori-motrices. In Fraisse Paul (dir.), Piaget Jean (dir), Traité de psychologie expérimentale. Tome II : Sensation et motricité (p. 125-171). Paris : Presses universitaires de France.

1971 - Leplat, J. La psychologie du travail. In M. Reuchlin, Traité de psychologie appliquée. 1, Les applications de la psychologie (p. 105-160). Paris: Presses universitaires de France.

1972 - Leplat, J. La psychologie du travail en ergonomie. In M. Reuchlin (dir.), Traité de psychologie appliquée. 3, Travailleurs et systèmes techniques (p. 61-136). Paris : Presses universitaires de France.

1973 - Leplat, J. La méthode expérimentale en psychologie appliquée. In M. Reuchlin (dir.), Traité de psychologie appliquée. 2, Les méthodes de la psychologie appliquée (p. 19-61). Paris : Presses universitaires de France.

411980 - Leplat, J. Développement et dégradation des habiletés dans le travail. In Société française de psychologie, Équilibre ou fatigue par le travail ? (p. 55-63). Paris: Entreprise moderne d'édition.

21982 - Leplat, J. Les situations de travail, terrains d'avenir pour la psychologie. In P. Fraisse (dir.), Psychologie de demain (p. 311-330). Paris: Presses universitaires de France. 

Fundaçao Getulio Vargas. française. Octarès. Complutense.

1989 - Leplat, J. Quelle place pour l'erreur humaine et pour la fiabilité humaine en ergonomie. In Anais, Actes du $4^{\text {ème }}$ Seminario Brasileiro de ergonomia. Rio de Janeiro :

1990 - Leplat, J. About Psychological Taxonomies. In J. Rasmussen, B. Brehmer, M. de Montmollin, J. Leplat, J. (éd.). Taxonomy for analysis of work domains. Proceedings of the first MOHAWC workshop, Liège, May 15-16, 1990. Tome 2 (p. 1-18). Roskilde, Denmark : Risø National Laboratory.

1991 - Leplat, J. Compétence et ergonomie. In R. Amalberti, M. de Montmollin, J. Theureau. Modèles en analyse du travail (p. 295-316). Liège : Pierre Mardaga.

1992 - Leplat, J. Conférence introductive. In M. Neboit, É. Fadier (éd.). Facteurs humains de la fiabilité et de la sécurité des systèmes complexes : actes du colloque tenu à Vandoeuvre les 17 et 18 avril 1991. Nancy : INRS.

1992 - Leplat, J. Les aspects cognitifs dans la formation. In F. Ginsbourger (coord.), V. Merle (coord.), G. Vergnaud (coord.), Formation et apprentissage des adultes peu qualifiés : actes du colloque des 24 et 25 juin 1992 (p. 87-116.). Paris : La Documentation

1993 - Leplat, J. Ergonomie et activités collectives. In F. Six, X. Vaxevanoglou (dir.). Les aspects collectifs du travial : actes du XXVIIème congrès de la Société d'ergonomie de langue française, Lille, 23-25 septembre 1992 (p. 7-27). Toulouse : Octarès.

1995 - Leplat, J. Préface. In P. Rabardel. Les hommes et les technologies : approche cognitive des instruments contemporains (p. 5-7). Paris : Armand Colin.

1996 - Leplat, J. Quelques aspects de la complexité en ergonomie. In F. Daniellou (dir.), L'ergonomie en quête de ses principes : débats épistémologiques (p. 57-76). Toulouse :

1996 - Leplat, J. Petites histoires pour des Histoires. In Y. Clot. Les histoires de la psychologie du travail : approche pluri-disciplinaire (p. 87-98). Toulouse : Octarès.

1997 - Leplat, J. Les lectures des ergonomes. In Recherche, pratique, formation en ergonomie: évolutions et interactions dans le contexte social, économique et technique : actes du XXXIIème congrès de la Société d'ergonomie de langue française (p. 735-742). Bron : Éditions Guerra.

1997 - Leplat, J. Event analysis and responsibility in complex systems. In A. Hale, B. Wilpert, M. Freitag (eds). After the event (p. 23-40). Oxford : Pergamon.

1998 - Leplat, J. \& Cuny, X. Las condiciones del trabajo. In J. J. Castillo, J. Villena (eds). Ergonomia : conceptos y methodos (p. 109-124). Madrid : Editorial Complutense.

51998 - Leplat, J. \& Hoc, J.-M. Tarea y actividad en el andisis psychologico de situaciones. In J. J. Castillo, J. Villena (eds). Ergonomia : conceptos y methodos (p. 163-180). Madrid : Editorial Complutense.

1998 - Leplat, J. Las representaciones funcionales en el trabajo. In J. J. Castillo, J. Villena (eds). Ergonomia: conceptos y methodos (p. 181-196). Madrid: Editorial

571999 - Leplat, J. Préface. In D. R. Kouabenan. Explication naïve de l'accident et prévention (p. 3). Toulouse : Octarès. 
2000 - Leplat, J. L'environnement de l'action en situation de travail. In Centre de recherche formation, Conservatoire national des arts et métiers, L'analyse de la singularité de l'action (p. 107-132). Paris : Presses universitaires de France.

2000 - Leplat, J. La gestion des communications par le contexte. In B. Mélier (coord.), Y. Quéinnec (coord.). Communication et travail: actes du 35ème congrès de la Self., Toulouse, les 20,21 et 22 septembre 2000 (p. 36-43). Toulouse : Octarès.

2002 - Terssac G. de \& Leplat, J. La fiabilité et l'ergonomie : spécificité et complémentarité. In Terssac Gilbert de, Le travail : une aventure collective : recueil de textes (p. 93-101). Toulouse : Octarès, (Coll. Travail et activité humaine)

2002 - Leplat, J. Éléments pour une histoire de la notion de charge mentale. In M. Jourdan, J. Theureau (éd.). Charge mentale : notion floue et vrai problème (p. 27-40). Toulouse : Octarès.

2003 - Leplat, J. Questions autour de la notion de risque. In D.R. Kouabenan, M. Dubois (ed.). Les risques professionnels : évolutions des approaches, Nouvelles perspectives (p. 37-52). Toulouse : Octarès.

2003 - Leplat, J. La modélisation en ergonomie à travers son histoire. In J.-C. Spérandio, M. Wolff (éd.). Formalismes de modélisation pour l'analyse du travail et l'ergonomie (p. 1-26). Paris : Presses universitaires de France.

2003 - Leplat, J. Préface. In C. Martin, D. Baradat (éd.). Des pratiques en réflexion (p. 1-6). Toulouse : Octarès.

2004 - Leplat, J. \& Montmollin, M. de. Les voisinages disciplinaires de l'ergonomie. In P. Falzon (dir), Ergonomie (p. 51-65). Paris : Presses universitaires de France.

2005 - Leplat, J. Préface. In P. Pastré (dir.). Apprendre par la simulation : de l'analyse du travail aux apprentissages professionnels (p. 1-6). Toulouse : Octarès.

2006 - Leplat, J. 15 ans d'analyse de l'activité : quelles évolutions ? In G. Valléry (dir.), R. Amalberti (dir.), L'analyse du travail en perspectives : influences et évolutions (p. 17-31). Toulouse : Octarès, (Coll. Le travail en débats/Entreprise, travail, emploi)

2006 - Leplat, J. Risque et perception du risque dans l'activité. In D.R. Kouabenan, B. Cadet, D. Herman, M.T. Munoz Sastre (ed.). Psychologie du risqué (p. 21-33). Bruxelles : De Bœck.

692013 - Leplat, J. Préface. In A. Drouin (coord.). Ergonomie : travail, conception, santé : cinquantenaire de la Société d'ergonomie de langue française, 1963-2013 (p. 1-2). Toulouse : Octarès.

02014 - Leplat, J. Aperçu sur les critères à la lumière de l'histoire. In D. Lhuillier (coord.). Qualité du travail, qualité au travail (p. 17-25). Toulouse : Octarès.

2015 - Leplat, J. L'analyse du travail : deux auteurs, une histoire, une actualité. In R. Ouvrier-Bonnaz, A. Weill-Fassina (coord.). André Ombredane (1898-1958), Jean-Marie Faverge (1912-1988) : l'analyse du travail, ruptures et évolutions (p. 55-64). Toulouse : Octarès.

2017 - Leplat, J. Concepts et méthodologie d'intervention : quelques aperçus historiques. In A.-L. Ulmann (dir.), A. Weill-Fassina (dir.), T. H. Benchekroun (dir.), Intervenir : histoires, recherches, pratiques (p. 15-24). Toulouse : Octarès, (Coll. Travail et activité humaine) 
2018 - Leplat, J. Jean-Marie Faverge et Antoine Léon: cheminement et rencontre autour de l'analyse du travail. In S. Blanchard, R. Ouvrier-Bonnaz (coord.). Connaissance du travail et orientation: une histoire en débats (p. 93-97). Toulouse : Octarès.

\section{Artigos} festion professionnelle existant au sein des entreprises = Investigation of technical training programs existing in industries. Bulletin du Centre d'études et recherches psychotechniques, 3, pp. 235-324. few critical works on time-motion
psychotechniques, 3, pp. 351-368. 
1957 - Browaeys, R. \& Leplat, J. Étude d'un travail de surveillance = Study of a work of inspection. Bulletin du Centre d'études et recherches psychotechniques, 6, pp. 373-378. 1959 - Leplat, J. \& Rouanet, H. Décisions et fonctions d'utilité = Decision-making and functions of utility. Bulletin du Centre d'études et recherches psychotechniques, 8, pp. 122-128.

1961 - Leplat, J. Psychologie expérimentale et étude des accidents = Experimental psychology and the study of accidents. Bulletin du CERP, 10(4), pp. 473-487.

1962 - Leplat, J. Psychologie et aménagement du travail. Le Travail humain, 25(3/4), pp. 269-280.

1962 - Leplat, J. Dispersion des signaux et niveau de vigilance. L'Année psychologique, 62(1), pp. 17-28. http://dx.doi.org/10.3406/psy.1962.7154

1963 - Leplat, J., Lantier, F., Barbichon, G. \& Grisez, J. Les recherches du Centre d'études et recherches psychotechniques en matière de formation professionnelle. Sociologie du travail, 5(4), pp. 411-415. https://dx.doi.org/10.3406/sotra.1963.1163

1964 - Leplat, J. Rôle des intervalles temporels entre signaux dans un travail de surveillance. L'Année psychologique, 64(2), pp. 353-374. http://dx.doi.org/10.3406/psy. 1964.27252

1967 - Leplat, J \& Sperandio, J.-C. La mesure de la charge de travail par la technique de la tâche ajoutée. L'Année psychologique, 67(1), pp. 255-277.

http://dx.doi.org/10.3406/psy.1967.27563

1967 - Leplat, J. Economics and Professional Training. Bulletin de psychologie, 20(18-19), pp. 1153-1163.

1967 - Leplat, J. Ergonomics and Professional Training: Work Analysis and Problem Diagnosis. Bulletin de psychologie, 20(16-17), pp. 1005-1012.

1967 - Leplat, J. Temporal Uncertainty and Additional Task. Bulletin du CERP, 16(1), pp. 41-59.

1970 - Leplat, J. Definition of the study object in the psychology of work. Bulletin de psychologie, 23(11-12), pp. 677-681.

1971 - Leplat, J., Chesnais, M. \& Gulian, E. Étude d'un modèle stochastique pour une tâche d'inspection. Le Travail humain, 34(2), pp. 265-276.

1971 - Leplat, J. (1971). Planning for action and regulation of a complex system. Bulletin de psychologie, 25(10-11), pp. 533-538.

1972 - Leplat, J. \& Pailhous, J. A taxonomy of tasks for a psychology of work : Some introductory considerations. Bulletin de psychologie, 25(10-11), pp. 539-545.

1973 - Leplat, J. \& Chesnais, M. Le contrôle des produits industriels : travaux actuels et perspectives d'étude. Le Travail humain, 36(1), pp. 75-94.

1973 - Leplat, J. \& Pailhous, J. Intellectual activity in work on an instrument. Bulletin de psychologie, 26(12-13), pp. 673-680.

1974 - Leplat, J. \& Pailhous, J. Comments on the origin of errors. Bulletin de psychologie, 27(13-14), pp. 729-736.

1974 - Leplat, J. Les critères dans les études ergonomiques de sécurité routière. Ergonomics, 17(5), pp. 663-675. 
1071975 - Leplat, J., Pailhous, J. \& Vermersch, P. Can the acquisition of a system of representation be rationalized ? Bulletin de psychologie, 28(7-8), pp. 398-409. 1975 - Pailhous, J., Chesnais, M. \& Leplat, J. Détection et localisation de lacunes en présentation tachistoscopique : rôle des visions fovéale et périphérique. L'Année psychologique, 75(2), pp. 445-456. http://dx.doi.org/10.3406/psy.1975.28107 1975 - Pailhous, J., Chesnais, M. \& Leplat, J. Détection et localisation de lacunes en présentation tachistoscopique: rôle des visions fovéale et périphérique. L'Année psychologique, 75(2), pp. 445-456. http://dx.doi.org/10.3406/psy.1975.28107

1101976 - Leplat, J. Study of hypotheses in natural environmental settings. Bulletin de psychologie, 30(1-2), pp. 25-29.

1111976 - Leplat, J. \& Pailhous, J. Cognitive conditions of the acquisition and use of sensorimotor skills. Bulletin de psychologie, 29(4-7), pp. 205-211. 1977 - Leplat, J. Les facteurs déterminant la charge de travail : rapport introductif. Le Travail humain, 40(2), pp. 195-202.

1131977 - Leplat, J. \& Welford, A. T. Communications présentées à un symposium du XXIe Congrès international de psychologie Paris, juillet 1976. Le Travail humain, 40(2), pp. 193.

1141978 - Leplat, J. IIe congrès de la société de biomécanique (Liège, 22 et 23 septembre 1977). Le Travail humain, 41(1), pp. 185-190. 1978 - Leplat, J. L'équivalence des situations de laboratoire et de terrain. Le Travail humain, 41(2), pp. 307-318. 1978 - Leplat, J. Factors Determining Work-load. Ergonomics, 21(3), pp. 143-149. 1978 - Leplat, J. Régulation de l'action et connaissance des résultats. Psychologie et éducation (revue de recherche du laboratoire associé au. CNRS), 259(2), pp. 73-79. 1978 - Leplat, J. \& Pailhous, J. Task description : Status and role in problem solving. Bulletin de psychologie, 31(1-2), pp. 149-156. 1980 - Leplat, J. Overview of the psychology of work. Bulletin de psychologie, 33(4-11), pp. 195-200. 1981 - Leplat, J. \& Pailhous, J. L'acquisition des habiletés mentales : la place des techniques. Le Travail humain, 44(2), pp. 275-282. 1981 - Leplat, J. \& Hoc, J.-M. Subsequent verbalization in the study of cognitive processes. Ergonomics, 24(10), pp. 743-755. 1982 - Leplat, J. Fiabilité et sécurité. Le Travail humain, 45(1), pp. 101-108. 1983 - Leplat, J. Statut et fonction des communications dans l'activité des équipes de travail. Psychologie française, 28(3/4), pp. 247-253. 1983 - Leplat, J. \& Hoc, J.M. Tâche et activité dans l'analyse psychologique des situations. Cahiers de psychologie cognitive, 3. 1984 - Leplat, J. \& Savoyant, A. Ordonnancement et coordination des activités dans les travaux individuels et collectifs. Bulletin de psychologie, 37(6-10), pp. 271-278. 1984 - Montmollin, M., de \& Leplat, J. Les cadres travaillent-ils ? : à propos d'un ouvrage de Luc Boltansky. Le Travail humain, 47(1), pp. 89-93. 1985 - Leplat, J. La psychologie du travail : recherches et inventions. Psychologie française, 30(2), pp. 163-167. 

36(1), pp. 9-27. 32(7), pp. 813-822. pp. 27-31. 39(2), pp. 143-154. 57(3), pp. 209-226. pp. 101-114. psychologie, 39(1), pp. 9-24. pp. 189-204. 49(1), pp. 31-42.

1985 - Leplat, J. Les représentations fonctionnelles dans le travail. Psychologie française, 30(3/4), pp. 269-275.

1986 - Leplat, J. L'analyse psychologique du travail. Revue de psychologie appliquée,

1988 - Leplat, J. L'analyse de l'erreur dans les nouvelles technologies: voies de recherche. Revue de psychologie appliquée, 38(2), pp. 151-160.

1988 - Leplat, J. \& Bellagh, M. A. Des recommandations utiles pour les psychologues du travail maghrébins (entretien). Horizons Maghrébins - Le droit à la mémoire, 12(1), pp. 27-30. http://dx.doi.org/10.3406/horma.1988.1473

1989 - Leplat, J. \& Bertrand, L. Rôle du schéma dans un diagnostic de panne sur une motrice de métro. Performances, 41, pp. 16-25.

1989 - Leplat, J. Error analysis, instrument and object of task analysis. Ergonomics,

1990 - Leplat, J. La fiabilité et l'ergonomie : spécificité et complémentarité. Revue de psychologie appliquée, 40(3), pp. 377-386.

1990 - Leplat, J. \& Terssac, G. de. Facteurs humains de la fiabilité. Performances, 47,

1990 - Leplat, J. Relations between task and activity: elements for elaborating a framework for error analysis. Ergonomics, 33(10/11), pp. 1389-1402.

1990 - Leplat, J \& Terssac, G. de. Reliability and ergonomics: specificity and complementarity. Revue de psychologie appliquée, 40(3), pp. 377-386.

1990 - Leplat, J. Skills and tacit skills : a psychological perspective. Applied psychology,

1993 - Leplat, J. L'analyse psychologique du travail : quelques jalons historiques. Le Travail humain, 56(2/3), pp. 115-131.

1993 - Leplat, J. Intention and error : a contribution to a study of responsibility. Revue européenne de psychologie appliquée, 43(4), pp. 279-287.

1994 - Leplat, J. Collective activity in work : some lines of research. Le Travail humain,

1995 - Leplat, J. À propos des compétences incorporées. Éducation permanente, 123,

1995 - Leplat, J. Cause et risque dans l'analyse des accidents. Revue romaine de

1998 - Leplat, J. À propos des procédures. Performances, 94, pp. 6-15.

1998 - Leplat, J. About implementation of safety rules. Safety Science, 29(3), pp. 189-204. http://dx.doi.org/10.1016/S0925-7535(98)00022-8

1998 - Leplat, J. \& Hale, A. Guest editorial : Safety rules. Safety Science, 29, pp. 159-161.

1998 - Leplat, J. About implementation of safety rules. Safety Science, 29(3),

1999 - Leplat, J. Cognitive error analysis. Revue européenne de psychologie appliquée, 
2000 - Leplat, J. La psychologie du travail $=$ The psychology of work. Psychologie française, 45(1), pp. 83-96.

2000 - Leplat, J. Compétences individuelles, compétences collectives. Psychologie du travail et des organisations, 6(3-4), pp. 47-73.

2001 - Leplat, J. La gestion des communications par le contexte. Perspectives interdisciplinaires sur le travail et la santé, 3(1). http://dx.doi.org/10.4000/pistes.3755

2002 - Leplat, J. De l'étude de cas à l'analyse de l'activité. Perspectives interdisciplinaires sur le travail et la santé, 4(2). http://dx.doi.org/10.4000/pistes.3658

2004 - Gadbois, C. \& Leplat, J. Connaissances et interventions. Activités, 1(1), pp. 6-22. http://dx.doi.org/10.4000/activites.1138

2004 - Leplat, J. Éléments pour l'étude des documents prescripteurs. Activités, 1(2). http://dx.doi.org/10.4000/activites.1293

2004 - Leplat, J. L'analyse psychologique du travail. Revue Européenne de Psychologie Appliquée/European Review of Applied Psychology, 54(2), pp.101-108. http:// dx.doi.org/10.1016/j.erap.2003.12.006

2005 - Clot, Y. \& Leplat, J. La méthode clinique en ergonomie et en psychologie du travail. Le Travail humain, 68(4), pp. 289-316. http://dx.doi.org/doi:10.3917/th.684.0289

2005 - Leplat, J. Les automatismes dans l'activité : pour une réhabilitation et un bon usage. Activités, 2(2). http://dx.doi.org/10.4000/activites.1797

2006 - Leplat, J. La notion de régulation dans l'analyse de l'activité. Perspectives interdisciplinaires sur le travail et la santé, 8(1). http://dx.doi.org/10.4000/pistes.3101

2006 - Leplat, J. Les contextes en formation. Éducation permanente, 166, pp. 29-48.

2008 - Leplat, J. Formation et didactique professionnelles : un chemin psychologique dans l'histoire. Travail et apprentissages, 1, pp. 22-33.

2008 - Leplat, J. Les compétences dans l'activité et leur analyse. Psihologia Resurselor Umane, 6(2), pp. 16-30. http://pru.apio.ro/index.php/prujournal/article/view/188

2009 - Leplat, J. L'erreur comme défaut et moyen de contrôle de l'activité en situation de travail $=$ The error as a defect and as a mean of controlling the activity in work situation. Psihologia Resurselor Umane Revista AsociaŢiei de Psihologie Indusstrială şi Organizaţională, 7(1), pp. 14-28.

2010 - Leplat, J. Jalons dans l'œuvre d'Alain Savoyant. Travail et apprentissages, 5, pp. 13-30.

2011 - Rogalski, J. \& Leplat, J. L'expérience professionnelle : expériences sédimentées et expériences épisodiques. Activités, 8(2). http://dx.doi.org/10.4000/activites.2556

2011 - Leplat, J. Comentários ao artigo "Contributo para uma introdução ao estudo dos sistemas homens-máquinas" de Michel Olivier. Laboreal, 7, (2), pp. 69-72. http:// laboreal.up.pt/revista/artigo.php?id=37t45nSU547112435:2573:4791

2013 - Leplat, J. Les gestes dans l'activité en situation de travail : aperçu de quelques problèmes d'analyse. Perspectives interdisciplinaires sur le travail et la santé, 15(1). http://dx.doi.org/10.4000/pistes.2951

2013 - Leplat, J. Contribution à une introduction aux études des systèmes hommesmachine de Michel Olivier, Pistes, 15(3), 5 p. https://journals.openedition.org/pistes/ 3497 
Notas de leitura 189-191. 307-312. 101-103. pistes/3762 pistes/3756 pistes/3764 pistes/3791

2015 - Leplat, J. Um modelo de investigação coletiva e internacional acerca da segurança. Laboreal, 11(1), pp. 106-108. http://dx.doi.org/10.15667/laborealxi0115jl

2016 - Leplat, J. Maurice Reuchlin e a dialética laboratório / terreno. Laboreal, 12(1), pp. 93-97. http://dx.doi.org/10.15667/laborealxii0116jl

2018 - Leplat, J. Um retorno a Gilbert Simondon e à sua obra inicial. Laboreal, 14(1), pp. 65-68. http://dx.doi.org/10.15667/LABOREALXIV0118JL

1990 - Leplat, J., \& Hoc, J.-M. (1990). Notes de lecture. Le Travail Humain, 53(2),

1991 - Leplat, J. Notes de lecture. Le Travail Humain, 54(4), 391-392.

1991 - Leplat, J. Notes de lecture. Le Travail Humain, 54(3), 283-286.

1992 - Leplat, J., Hoc, J.-M., \& Rogalski, J. Notes de lecture. Le Travail Humain, 55(3),

1993 - Wisner, A., Leplat, J., \& Hoc, J.-M. Notes de lecture. Le Travail Humain, 56(1),

2001 - Leplat, J. La signalétique : conception, validation, usages de Cambon de Lavalette, Doré et Tijus. Perspectives interdisciplinaires sur le travail et la santé, (3-2). http://journals.openedition.org/pistes/3794

2001 - Leplat, J. Proactive risk management in a dynamic society de Rasmussen et Syedung. Perspectives interdisciplinaires sur le travail et la santé, (3-2). http:// journals.openedition.org/pistes/3793

2002 - Leplat, J. Apprentissage organisationnel. Théorie, méthode, pratique de Argyris et Schön. Perspectives interdisciplinaires sur le travail et la santé, (4-1). http:// journals.openedition.org/pistes/3758

2002 - Leplat, J. De la motivation à la formation de Carré. Perspectives interdisciplinaires sur le travail et la santé, (4-1). http://journals.openedition.org/

2002 - Leplat, J. Discours sur l'organisation du travail de De Montmollin. Perspectives interdisciplinaires sur le travail et la santé, (4-1). http://journals.openedition.org/

2002 - Leplat, J. Error prevention and well-being at work in western Europe and Russia. Psychological traditions and new trends de De Keyser et Leonova. Perspectives interdisciplinaires sur le travail et la santé, (4-1). http://journals.openedition.org/

2002 - Leplat, J. Le travail : une activité collective de De Terssac. Perspectives interdisciplinaires sur le travail et la santé, (4-2). http://journals.openedition.org/

2002 - Leplat, J. L'intervention ergonomique. Un regard sur la pratique professionnelle de Lamonde. Perspectives interdisciplinaires sur le travail et la santé, (4-1). http:// journals.openedition.org/pistes/3768

2002 - Leplat, J. Management des compétences. Réalisations, concepts, analyses de Aubret, Gilbert et Pigeyre. Perspectives interdisciplinaires sur le travail et la santé, (4-2). http://journals.openedition.org/pistes/3787 

pistes $/ 3780$ pistes/3783 pistes/3248

2002 - Leplat, J. Organiser la fiabilité de Bourrier. Perspectives interdisciplinaires sur le travail et la santé, (4-1). http://journals.openedition.org/pistes/3760

2002 - Leplat, J. Psychologie du travail de Karnas. Perspectives interdisciplinaires sur le travail et la santé, (4-2). http://journals.openedition.org/pistes/3789

2002 - Leplat, J. Task analysis d'Annett et Stanton. Perspectives interdisciplinaires sur le travail et la santé, (4-1). http://journals.openedition.org/pistes/3766

2002 - Leplat, J. Work process knowledge de Boreham, Samurçay et Fischer. Perspectives interdisciplinaires sur le travail et la santé, (4-2). http:// journals.openedition.org/pistes/3785

2003 - Leplat, J. Le travail collectif dans l'industrie à risque. Six points de vue de chercheurs étayés et discutés de Maggi et Lagrange. Perspectives interdisciplinaires sur le travail et la santé, (5-1). http://journals.openedition.org/pistes/3781

2003 - Leplat, J. Lifelong learning : which ways forward ? de Colardyn. Perspectives interdisciplinaires sur le travail et la santé, (5-1). http://journals.openedition.org/

2003 - Leplat, J. Stress au travail et santé psychique de Neboit et Vézina. Perspectives interdisciplinaires sur le travail et la santé, (5-1). http://journals.openedition.org/

2004 - Leplat, J. Recherches en didactique professionnelle de Samurçay et Pastré. Perspectives interdisciplinaires sur le travail et la santé, (6-2). http:// journals.openedition.org/pistes/3259

2005 - Leplat, J. Handbook of cognitive task design de Hollnagel. Perspectives interdisciplinaires sur le travail et la santé, (7-1). http://journals.openedition.org/

2005 - Leplat, J. Le facteur humain. Réinventer notre rapport à la technologie de Vicente. Perspectives interdisciplinaires sur le travail et la santé, (7-3). http:// journals.openedition.org/pistes/3137

2005 - Leplat, J. Les savoirs d'action : une mise en mots des compétences ? de Barbier et Galatanu. Perspectives interdisciplinaires sur le travail et la santé, (7-1). http:// journals.openedition.org/pistes/3244

2005 - Leplat, Jacques. (2005). Modèles et méthodologies d'analyse des compétences. Le double éclairage des pratiques et des recherches de Hajjar et Baubion-Broye. Perspectives interdisciplinaires sur le travail et la santé, (7-3). http:// journals.openedition.org/pistes/3149

2005 - Leplat, J. Hoc, J.-M. \& Darses, F. (Eds.). (2004). Psychologie ergonomique : tendances actuelles. Paris : PUF, Collection Le Travail humain. 260 pages. Activités, 2(1). https://journals.openedition.org/activites/1635

2005 - Leplat, J. Bronckart, J.-P. (Ed.), Bulea, E., Filliettaz, L., Fristalon, I., Plazaola Giger, I. \& Revaz F. (2004). Agir et discours en situation de travail. Cahiers de la section des sciences de l'éducation, $\mathrm{n}^{\circ} 103$. Université de Genève (312 p.). Activités, 2(1). https://journals.openedition.org/activites/1628

2005 - Leplat, J. Alamargot, D., Terrier, P. \& Cellier, J.-M. (Eds). (2005). Production, compréhension et usages des écrits techniques au travail. Toulouse : Octarès Éditons, 243 pages. Activités, 2(2). https://journals.openedition.org/activites/1828 

pistes/3019 pistes/3776 activites/1973

2005 - Leplat, J. Norros, L. (2004). Acting under uncertainty. The core-task analysis in ecological study of work. VTT Industrial Systems, Techniicantie 12, P.O. Box, 1301, FIN-02044 VTT Finland. Activités, 2(2). https://journals.openedition.org/activites/1832 2006 - Leplat, J. Barriers and accident prevention de Hollnagel. Perspectives interdisciplinaires sur le travail et la santé, (8-2). http://journals.openedition.org/

2006 - Leplat, J. L'analyse des actions et des discours en situation de travail de Fillietaz et Bronckart. Perspectives interdisciplinaires sur le travail et la santé, (8-1). http:// journals.openedition.org/pistes/3774

2006 - Leplat, J. L'ergonomie de Darses et de Montmollin. Perspectives interdisciplinaires sur le travail et la santé, (8-1). http://journals.openedition.org/

2006 - Leplat, J. Rabardel, P. \& Pastré, P. (s/d). (2005). Modèles du sujet pour la conception. Dialectiques activités développement. Toulouse: Octarès. 260 pages. Activités, 3(1). https://journals.openedition.org/activites/1885

2007 - Leplat, J. La décision médicale collective. Pour des médecins moins savants et moins autonomes ? De Mollo et Sauvagnac. Perspectives interdisciplinaires sur le travail et la santé, (9-1). http://journals.openedition.org/pistes/3012

2007 - Leplat, J. Pratiques d'information-conseil en VAE (Validation des Acquis de l'Expérience). Une analyse des pratiques par le réseau des conseillers PRC (Point Relais Conseil) en Bourgogne de Mayen et Perrier. Perspectives interdisciplinaires sur le travail et la santé, (9-1). http://journals.openedition.org/pistes/3011

2007 - Leplat, J. Resilience engineering. Concepts and precepts de Hollnagel, Woods et Leveson. Perspectives interdisciplinaires sur le travail et la santé, (9-2). http:// journals.openedition.org/pistes/3770

2008 - Leplat, J. La conception en design industriel et en architecture. Désir, pertinence, coopération et cognition de Lebahar. Perspectives interdisciplinaires sur le travail et la santé, (10-2). http://journals.openedition.org/pistes/2927

2008 - Leplat, J. Travail et pouvoir d'agir de Clot. Perspectives interdisciplinaires sur le travail et la santé, (10-2). http://journals.openedition.org/pistes/2922

2008 - Leplat, J. Nosulenko, V. \& Rabardel, P. (2007). Rubinstein aujourd'hui. Nouvelles figures de l'activité humaine. Activités, 5(1). https://journals.openedition.org/

2008 - Leplat, J. Merri, M. (Ed.) (2007). Activité humaine et conceptualisation. Questions à Gérard Vergnaud. Toulouse: Presses universitaires du Mirail. Activités, 5(2). https://journals.openedition.org/activites/2057

2009 - Leplat, J. Remaining sensitive to the possibility of failure de Hollnagel, Nemeth et Dekker. Perspectives interdisciplinaires sur le travail et la santé, (11-1). http:// journals.openedition.org/pistes/2898

42009 - Leplat, J. Hollnagel, E. (2009). The ETTO Principle : Efficiency - Thoroughness Trade - Off. Why things that go right sometimes go wrong ?. Farnham (U.K.) : Ashgate. Activités, 6(2). https://journals.openedition.org/activites/2284 
2010 - Leplat, J. Owen, C., Béguin, P. \& Wackers, G. (2009). Risky word environment. Reappraising human work within fallible systems. Farnham (G.B.) : Ashgate. Activités, 7(2). https://journals.openedition.org/activites/2383

2011 - Leplat, J. Yvon, F. \& Saussez, F. (Eds) (2010). Analyser l'activité enseignante. Des outils méthodologiques et théoriques pour l'intervention et la formation. Laval (Canada): Les Presses de l'Université Laval. Activités, 8(1). https:// journals.openedition.org/activites/2532

2172011 - Leplat, J. Adé, D., \& De Saint-Georges, I. (Eds) (2010). Les objets dans la formation. Usages, rôles et significations. Toulouse : Octarès. Activités, 8(1). https:// journals.openedition.org/activites/2531

2182011 - Leplat, J. Pastré, P. (2011). La didactique professionnelle. Approche anthropologique $\mathrm{du}$ développement chez les adultes. Formation et pratiques professionnelles. Paris : PUF, 318 p. Activités, 8(2). https://journals.openedition.org/ activites $/ 2645$

2012 - Leplat, J. Norman, D.A., The design of futre things et Living with complexity. Activités, 9(1). https://journals.openedition.org/activites/184

2012 - Leplat, J. De Terssac, G., Mignard, J. Les paradoxes de la sécurité. Le cas d'AZF. Activités, 9(1). https://journals.openedition.org/activites/216

2212012 - Leplat, J. Molinié, A.-F., Gaudart, C. \& Pueyo, V. La vie professionnelle. Âge, expérience et santé à l'épreuve des conditions de travail. Activités, 9(2). https:// journals.openedition.org/activites/513

2222013 - Leplat, J. Contribution à une introduction aux études des systèmes hommesmachine de Michel Olivier. Perspectives interdisciplinaires sur le travail et la santé, (15-3). http://journals.openedition.org/pistes/3497

2013 - Leplat, J. La confiance au travail sous la coordination de Karsenty. Perspectives interdisciplinaires sur le travail et la santé, (15-3). http://journals.openedition.org/ pistes/3543

2013 - Leplat, J. (Se) Former pour transformer le travail. Dynamiques de constructions d'une analyse critique du travail. Sous la coordination de Catherine Teiger et Marianne Lacomblez. Perspectives interdisciplinaires sur le travail et la santé, (15-3). http:// journals.openedition.org/pistes/3499

2013 - Leplat, J. Vulnérabilités au travail. Naissance et actualité de la psychopathologie du travail de Veil, présentation par Dominique Lhuilier. Perspectives interdisciplinaires sur le travail et la santé, (15-1). http://journals.openedition.org/pistes/3216

2013 - Leplat, J. Datchary, C. La dispersion au travail. Activités, 10(2). https:// journals.openedition.org/activites/863

2014 - Leplat, J. Le risque et la règle. Le cas du bâtiment et des travaux publics de Cru. Perspectives interdisciplinaires sur le travail et la santé, (16-3). http:// journals.openedition.org/pistes/4014

2014 - Leplat, J. Le travail peut-il devenir supportable ? De Clot et Gollac. Perspectives interdisciplinaires sur le travail et la santé, (16-4). http://journals.openedition.org/ pistes/4314

2014 - Leplat, J. Falzon, P. (sous la direction de). (2013). Ergonomie constructive. Paris : PUF. Activités, 11(1). https://journals.openedition.org/activites/400 
2015 - Leplat, J. Mieux-être au travail : appropriation et reconnaissance de Bernoux. Perspectives interdisciplinaires sur le travail et la santé, (17-2). http:// journals.openedition.org/pistes/4532 2015 - Leplat, J. Mayen, P. \& Lainé, A. Apprendre à travailler avec le vivant ? Développement durable et didactique professionnelle. Activités, 12(1). https:// journals.openedition.org/activites/1052 2016 - Leplat, J. Dekker, S.W.A. Safety Differently: Human Factors for a New Era. Activités, 13(2). https://journals.openedition.org/activites/2898 2017 - Leplat, J. Dujarier, M.-A., Gaudart, C., Gillet, A. \& Lénel, P. L'activité en théories. Regards croisés sur le travail. Activités, 14(1). https://journals.openedition.org/ activites/2946

2342017 - Leplat, J. Christol-Souviron, M., Leduc, S., Drouin, A. \& Étienne, P. Performances humaines et techniques: d'hier VERS aujourd'hui. Activités, 14(1). https:// journals.openedition.org/activites/2960

\section{AUTORES}

\section{CORINNE LESPESSAILLES}

Centre de documentation sur la formation et le travail - Direction des bibliothèques et de la documentation - Conservatoire national des arts et métiers, 41 rue Gay Lussac, 75005 Paris (case courrier 4SCD01), France

corinne.lespessailles@lecnam.net

\section{CÉLINE LEVECQUE}

Bibliothèque Gay-Lussac

Direction des bibliothèques et de la documentation - Conservatoire national des arts et métiers, 41 rue Gay Lussac, 75005 Paris (case courrier 4SCD01), France celine.levecque@lecnam.net

\section{AURÉLIE PUYBONNIEUX}

Centre de documentation sur la formation et le travail - Direction des bibliothèques et de la documentation - Conservatoire national des arts et métiers, 41 rue Gay Lussac, 75005 Paris (case courrier 4SCD01), France aurelie.puybonnieux@lecnam.net

\section{AGATHE VUILLERMET}

Bibliothèque Gay-Lussac - Direction des bibliothèques et de la documentation - Conservatoire national des arts et métiers, 41 rue Gay Lussac, 75005 Paris, France agathe.vuillermet@lecnam.net 